\title{
\begin{tabular}{l|l} 
Mitraries & DSpace@MIT
\end{tabular}
}

\author{
MIT Open Access Articles
}

\section{Nitinol Stent Oversizing in Patients Undergoing Popliteal Artery Revascularization: A Finite Element Study}

The MIT Faculty has made this article openly available. Please share how this access benefits you. Your story matters.

Citation: Gökgöl, Can et al. “Nitinol Stent Oversizing in Patients Undergoing Popliteal Artery Revascularization: A Finite Element Study." Annals of Biomedical Engineering 43.12 (2015): 2868-2880.

As Published: http://dx.doi.org/10.1007/s10439-015-1358-8

Publisher: Springer US

Persistent URL: http://hdl.handle.net/1721.1/106573

Version: Author's final manuscript: final author's manuscript post peer review, without publisher's formatting or copy editing

Terms of Use: Article is made available in accordance with the publisher's policy and may be subject to US copyright law. Please refer to the publisher's site for terms of use. 


\title{
Nitinol Stent Oversizing in Patients Undergoing Popliteal Artery Revascularization: A Finite Element Study
}

\author{
Can Gökgöl, ${ }^{1}$ Nicolas Diehm, ${ }^{2,3}$ Farhad Rikhtegar Nezami, ${ }^{1,4}$ and Philippe Büchler ${ }^{1}$ \\ ${ }^{1}$ Institute for Surgical Technology \& Biomechanics, University of Bern, Bern, Switzerland; ${ }^{2}$ Clinical and Interventional \\ Angiology, Kantonsspital Aarau, Tellstrasse, Aarau 5001, Switzerland; ${ }^{3}$ University of Applied Sciences Furtwangen, Villingen- \\ Schwenningen, Germany; and ${ }^{4}$ Harvard-MIT Biomedical Engineering Center, Massachusetts Institute of Technology, \\ Cambridge, MA 02139, USA
}

(Received 31 March 2015; accepted 9 June 2015; published online 23 June 2015)

Associate Editor Estefanía Peña oversaw the review of this article.

\begin{abstract}
Nitinol stent oversizing is frequently performed in peripheral arteries to ensure a desirable lumen gain. However, the clinical effect of mis-sizing remains controversial. The goal of this study was to provide a better understanding of the structural and hemodynamic effects of Nitinol stent oversizing. Five patient-specific numerical models of noncalcified popliteal arteries were developed to simulate the deployment of Nitinol stents with oversizing ratios ranging from 1.1 to 1.8 . In addition to arterial biomechanics, computational fluid dynamics methods were adopted to simulate the physiological blood flow inside the stented arteries. Results showed that stent oversizing led to a limited increase in the acute lumen gain, albeit at the cost of a significant increase in arterial wall stresses. Furthermore, localized areas affected by low Wall Shear Stress increased with higher oversizing ratios. Stents were also negatively impacted by the procedure as their fatigue safety factors gradually decreased with oversizing. These adverse effects to both the artery walls and stents may create circumstances for restenosis. Although the ideal oversizing ratio is stentspecific, this study showed that Nitinol stent oversizing has a very small impact on the immediate lumen gain, which contradicts the clinical motivations of the procedure.
\end{abstract}

Keywords-Self-expanding Nitinol stents, Stent oversizing, Stent deployment, Popliteal arterial segment, Patient-specific arterial geometry, Curved arteries, Acute lumen gain, Finite element analysis (FEA), Computational fluid dynamics (CFD) analysis.

\section{INTRODUCTION}

Peripheral arterial disease (PAD) is commonly treated by endovascular means. ${ }^{10}$ Restenosis remains

Address correspondence to Nicolas Diehm, Clinical and Interventional Angiology, Kantonsspital Aarau, Tellstrasse, Aarau 5001, Switzerland. Electronic mail: nicolas.diehm@ksa.ch the major drawback of endovascular revascularization and may affect up to $70 \%$ of the patients undergoing plain balloon angioplasty of the femoro-popliteal (FP) arteries. ${ }^{30}$ The introduction of self-expanding Nitinol stents has reduced restenosis rates within these arterial segments to about $40 \%$ depending on arterial lesion morphology. ${ }^{31}$ Two main explanations have been proposed to explain the causes for restenosis subsequent to Nitinol stent placement; stent fractures ${ }^{29}$ and chronic irritation of arterial walls during or following stent deployment. ${ }^{11}$ While stent fractures may be explained by the high mechanical loads acting upon the stent during flexion of the leg, ${ }^{9}$ arterial wall damage may result from oversizing of Nitinol stents, which is frequently performed to achieve large luminal gain and to prevent stent migration. . $^{28,41}$

Several investigations have been performed to evaluate the effect of stent diameter on the risk of restenosis. ${ }^{1,16,22,28,41}$ A correlation between Nitinol stent oversizing and restenosis has been evaluated using animal models, for which oversized stents have been implanted in healthy arteries of Yucatan swines, ${ }^{41}$ mini-pigs,${ }^{28}$ canines ${ }^{1,16}$ and humans. ${ }^{22}$ Based on these studies, it is difficult to assess the clinical effect of Nitinol stent oversizing a few months following endovascular intervention. In some studies, stent oversizing was associated with adequate luminal gain immediately during the implantation, but ultimately led to excess neointimal hyperplasia and restenosis. ${ }^{1,28,41}$ Other authors reported limited restenosis months after implantation of oversized stents. ${ }^{16,22}$

A better description of the biomechanical implications of oversizing on arterial tissues may help to understand the mechanisms responsible for restenosis and therefore help the clinicians to select the 
appropriate stent size during intervention. There are various structural finite element (FE) analyses on stent deployment. ${ }^{5,6,20,24,39,40}$ Similarly, computational fluid dynamics (CFD) simulations have been widely used to investigate the hemodynamic properties of stented arteries and their implications for neointimal hyperplasia. ${ }^{3,4,15,18,23,33}$ However, the only numerical studies conducted on stent mis-sizing were found to be on balloon-expandable stents deployed in straight cylindrical models of healthy ${ }^{3,18}$ or calcified coronary arteries. ${ }^{37}$ Due to the significant differences in their deployment procedures, the results from these studies could not be adopted for self-expandable Nitinol stents. Thus, the aim of the present study was to quantify the biomechanical effects of Nitinol stent oversizing using numerical models of non-calcified popliteal arteries obtained from patients undergoing endovascular revascularization. Two main aspects were considered; the mechanical effect of the stent on arterial walls and hemodynamic alterations induced by stents of varying size. We hypothesized that wall stresses increase with increasing stent size, which may lead to chronic arterial wall damage. Moreover, we suggested that the presence of an arterial curvature would aggravate the effects of oversizing by further increasing arterial stress levels. Finally, we hypothesized that oversizing could trigger neointimal hyperplasia in the arterial walls by reducing wall shear stress (WSS) and by increasing oscillatory shear index (OSI).

\section{MATERIALS AND METHODS}

The anatomies of the arterial models were obtained from five patients ( 4 men and 1 woman; age, 56-79 years; mean age $69 \pm 9.1$ years) undergoing intra-arterial 3D rotational digital subtraction angiography (AXIOM-Artis, Siemens, Germany) prior to endovascular revascularization of their FP arteries. ${ }^{9}$ For four patients, the investigated segment of the arterial tract started from the descending genicular artery and ended at the superior genicular artery. For the remaining patient, the segment ended at the inferior genicular artery. Ethics committee approval had been obtained prior to the start of this investigation. Further information about the patients and the acquisition process could be found in a previous study. ${ }^{9}$

All 3D angiographic examinations were saved digitally and transferred to a workstation capable of $3 \mathrm{D}$ post-processing. Following the segmentation of the vasculatures on the $3 \mathrm{D}$ image datasets with an image analysis software (Amira 5.2; FEI Visualization Sciences Group, Burlington, MA, USA), arterial centerlines were extracted and shortened to only have the section with the maximal curvatures (mean length of
$53 \mathrm{~mm}$, with a range of $47-59.5 \mathrm{~mm}$ ). Each centerline was described by 108 points. For each patient, the FE mesh of the artery was reconstructed around these centerlines using a script in Matlab (The Mathworks Inc., Natick, MA, USA). For each node on the centerline, the script generated five rings, each with 128 nodes. This meshing approach resulted in the same number of nodes and elements for all the arterial models. Furthermore, for longer centerlines, the distance between the rings were adjusted so that the element size around the region of stent implantation would be the same as the shorter centerlines. Apart from the patient-specific arteries, a sixth arterial model was created from a straight centerline to represent a generic, straight artery. The arteries were assumed to have a circular cross section of constant diameter. Three concentric arterial layers were reconstructed; the intima $(5-5.3 \mathrm{~mm})$, the media $(5.3-5.9 \mathrm{~mm})$ and the adventitia $(5.9-6 \mathrm{~mm})$. All of the arterial models had 54,874 linear hexahedral reduced integration (C3D8R) elements; 13,696 elements in both intima and adventitia and 27,392 elements in the medial arterial layer. The validity of the mesh was confirmed through a mesh sensitivity analysis, in which the maximum circumferential stresses in each layer of the artery changed by $3 \%$ at the diastolic and systolic points of a cardiac cycle.

The material model adopted for the artery was an anisotropic, hyper-elastic model that represented two families of collagen fibers. ${ }^{8,12}$ The strain energy density function was based on the constitutive laws proposed by Gasser et al., which allows not only the specification of fiber orientations, but also the inclusion of fiber dispersions within each layer. ${ }^{8}$ The material parameters for the model were taken from experiments performed on non-calcified iliac arteries (Table 1). ${ }^{8,12}$ The two families of fibers were symmetrically arranged with respect to the arteries' circumferential directions. In accordance with previous findings, ${ }^{8}$ the fibers within media were perfectly aligned in circumferential direction $(\kappa=0)$; whereas the fibers within intima and adventitia were modeled as slightly $(\kappa=0.02)$ and highly dispersed $(\kappa=0.226)$, respectively.

The arterial models were implanted with two commercially available, self-expanding stents: Astron-Pulsar and Astron (Biotronik AG, Bülach, Switzerland). Both stents were made of the same shape memory Nitinol, whose material coefficients were provided by the manufacturer (Table 2). Nevertheless, the stents exhibited different mechanical behaviors due to their different designs, with the Astron having larger strut width, thickness and outer diameter $(119,245 \mu \mathrm{m}$ and $5 \mathrm{~mm}$, respectively) than the Astron-Pulsar stent (80, $155 \mu \mathrm{m}$ and $3.5 \mathrm{~mm}$, respectively). FE meshes of both stents were produced with 173,160 and 91,248 C3D8R 
TABLE 1. Material parameters for the individual layers of the artery; intima, media and adventitia.

\begin{tabular}{llll}
\hline & Adventitia & Media & Intima \\
\hline $\mathrm{C}_{10}(\mathrm{kPa})$ & 7.64 & 15 & 31 \\
$k_{1}(\mathrm{kPa})$ & 996.6 & 4 & 51 \\
$k_{2}$ & 524.6 & 2.3 & 1.1 \\
$\delta_{1}=\delta_{2}\left(^{\circ}\right)$ & 50 & 7 & 5 \\
$\kappa$ & 0.226 & 0 & 0.02 \\
\hline
\end{tabular}

TABLE 2. Material parameters for the Nitinol model.

\begin{tabular}{ll}
\hline Austenite elasticity $E_{\mathrm{A}}(\mathrm{MPa})$ & 65,000 \\
Austenite Poisson's ratio $v_{\mathrm{A}}$ & 0.33 \\
Martensite elasticity $E_{\mathrm{M}}(\mathrm{MPa})$ & 23,500 \\
Martensite Poisson's ratio $v_{\mathrm{M}}$ & 0.33 \\
Transformation strain $\varepsilon^{\mathrm{L}}$ & 0.046 \\
Loading $(\delta \sigma / \delta T)_{L}(\delta \sigma / \delta T)\left[\mathrm{MPa} T^{-1}\right]$ & 0 \\
Start of transformation loading $\sigma_{\mathrm{L}}^{\mathrm{S}}(\mathrm{MPa})$ & 465 \\
End of transformation loading $\sigma_{\mathrm{L}}^{\mathrm{E}}(\mathrm{MPa})$ & 535 \\
Reference temperature $T_{0}\left({ }^{\circ} \mathrm{C}\right)$ & 37 \\
Unloading $(\delta \sigma / \delta T)_{\mathrm{U}}(\delta \sigma / \delta T)$ & 0 \\
Start of transformation unloăding $\sigma_{\mathrm{U}}^{\mathrm{S}}(\mathrm{MPa})$ & 227 \\
End of transformation unloading $\sigma_{\mathrm{U}}^{\mathrm{E}}(\mathrm{MPa})$ & 187 \\
Start of transformation stress in compression $\sigma_{C L}^{S}(\mathrm{MPa})$ & 582 \\
Volumetric transformation strain $\varepsilon_{\mathrm{V}}^{\mathrm{L}}$ & 0.046 \\
\hline
\end{tabular}

elements for Astron-Pulsar and Astron, respectively. The number of elements used to mesh the stents were determined by sensitivity analyses, in which the maximum principal strain values changed less than $2.5 \%$ in the stents' crimped configurations.

Due to the discontinuities induced by the large number of complex contact interactions between several components of the model, the structural analyses were conducted with Abaqus/Explicit (Dassault Systemes, Simulia Corp., RI, USA). A semi-automatic stable time increment of $4 \times 10^{-6}$ was defined to control mass scaling. This increment ensured that the ratio of kinetic to internal energy in the system remained below $5 \%$ to avoid dynamic effects, which guaranteed that the analysis remained quasi-static. To leave the stents constraint free and let them find their own mechanical equilibriums, all displacement boundary conditions were applied onto the nodes of two rigid cylinders that were meshed with 4 node quadrilateral shell elements, with reduced integration (S4R). They surrounded the inner and outer surfaces of the stents and acted as expansion and crimp tools (Fig. 1). While the undeformed diameter of the expansion tool corresponded to the inner diameter of the Astron-Pulsar stent; the size of the crimp tool was sufficiently large so that its initial contact with the stents is established only after the latter reach their unconstrained diameters. The contact between the surfaces was defined as a frictionless, hard contact. Additionally, arteries were fixed at both ends with the exception of the local radial directions. A total of four cases were considered for each artery, where stents with unconstrained diameters of 5.5, 6, 7 and $9 \mathrm{~mm}$ were used.

Prior to the oversizing analyses, the validity of the arterial model had been evaluated. Rectangular strips of each individual arterial layer were subjected to uniaxial extension and their mechanical behaviors in axial and circumferential directions had been compared with published experimental data. ${ }^{13}$ Another evaluation of the proposed arterial model was performed by calculating the compliance of the artery. The compliance was calculated as the ratio of the maximum nominal strain to the maximum pressure change during a cardiac cycle and the numerically obtained value was compared with in vivo measurements. ${ }^{35}$ The precision of the models used to simulate the stents was also evaluated. The radial force generated by the stents was calculated while crimping the stents to a $1.8 \mathrm{~mm}$ diameter followed by an expansion to their unconstrained diameters of $9 \mathrm{~mm}$. The radial force was compared with experimental measurements provided by the stent manufacturer. Finally, in order to ensure a $5 \mathrm{~mm}$ lumen at a mean blood pressure of $120 \mathrm{mmHg}$, the arterial models were run through an iterative process, ${ }^{7}$ in which the arterial expansion was estimated under the target pressure. The unloaded arterial geometry obtained following the last iteration was considered as the stress-free configuration.

Accurate calculation of the deployment requires considering the complete stress-strain history of the Nitinol stents. Therefore, full stent deployment, involving the preconditioning of the stents, was performed for all cases (Fig. 1). In the first step, the only active contact in the model was between the inner surface of the stent and the outer surface of the expansion tool. By radially displacing each node of the rigid tool, the stent was expanded to its desired unconstrained diameter. At this stage, the stent was subjected to annealing; a procedure used to remove the existing stresses, while preserving the deformed shape. Subsequently, the previous contact pair of stentexpansion tool was replaced with a contact interaction between the outer-surface of the stent and the innersurface of the crimp tool. In the next step, the stent was crimped to its deployment diameter $(1.25 \mathrm{~mm}$ for Astron-Pulsar and $1.88 \mathrm{~mm}$ for the Astron stent) by applying displacements to the nodes of the crimp-tool. During this step, a uniform pressure load of $120 \mathrm{mmHg}$ was applied to the inner surface of the arteries to expand the lumen diameters to exactly $5 \mathrm{~mm}$, which corresponded to the average lumen diameter of healthy arteries in physiological blood 


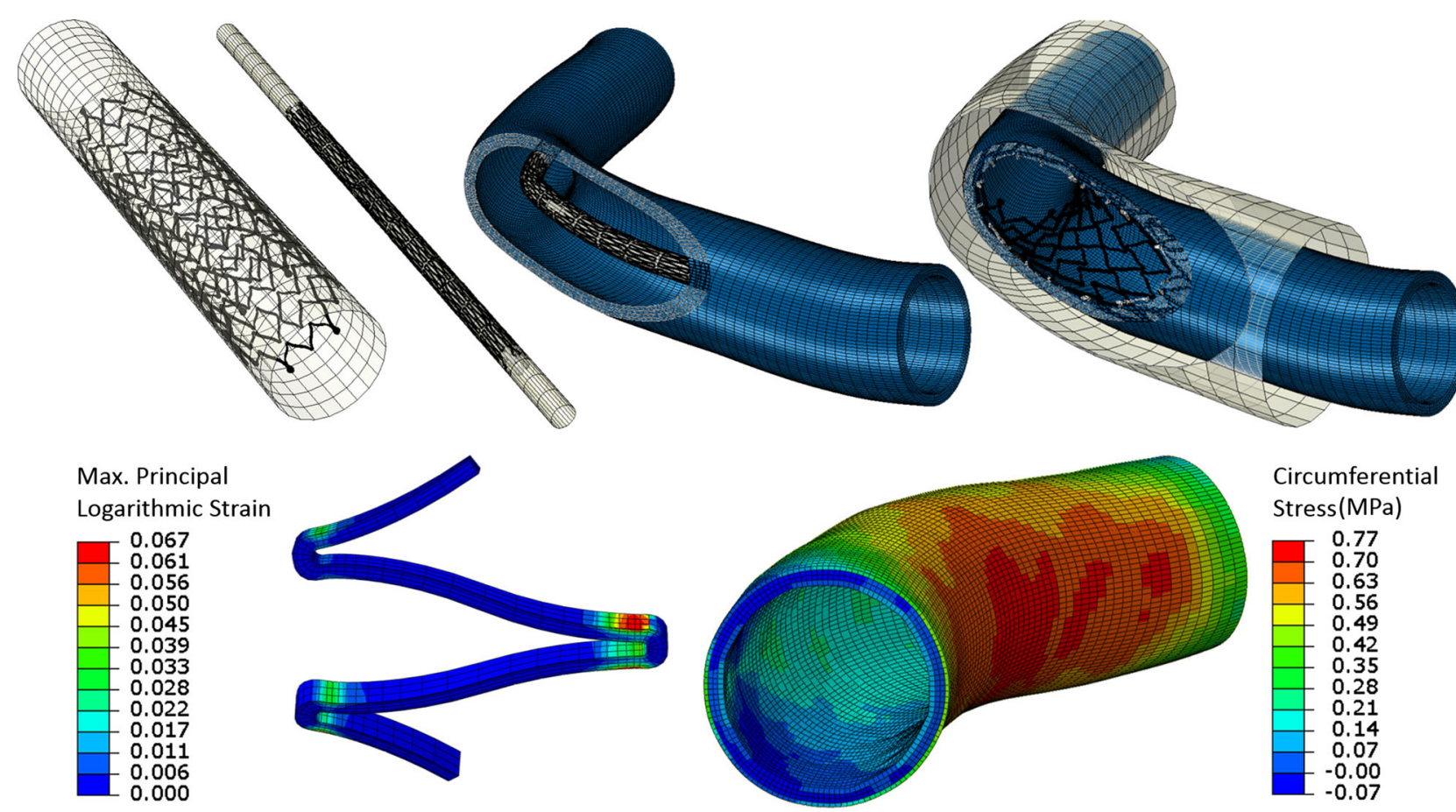

FIGURE 1. Example of the preconditioning and deployment of a Nitinol stent into a patient-specific artery. By radially displacing the nodes of the expansion tool (not shown), the Astron-Pulsar stent is expanded to its unconstrained diameter of $7 \mathrm{~mm}$ (top-left). After annealing, the stent is crimped to its deployment diameter of $1.2 \mathrm{~mm}$ by radially displacing the nodes of the crimp tool (topmiddle). The insertion of the stent into a patient-specific artery is achieved by a displacement-controlled deformation of the crimp tool into the shape of the arterial midline (top-middle). The crimp-tool is then expanded to its initial diameter, which results in the deployment of the stent as it detaches from the tool to contact the arterial walls (top-right). Following deployment, strains are concentrated at the outer sides of the struts (bottom-left) and stress distribution in different layers of the artery demonstrates the load-carrying behavior of the adventitia (bottom-right).

pressures. ${ }^{34}$ Following crimping, the crimp tool was deformed into the shape of the arterial centerline to simulate stent insertion. The contact pair was modified to include an interaction between the outer-surface of the stent with the inner-surface of the artery in addition to the existing stent-crimp tool pair. Consequently, during the tool's radial expansion to its final configuration (which depends on the unconstrained stent diameter for each oversizing case), the stent smoothly detached from the tool when it came into contact with the inner wall of the artery. After deployment, a cyclic blood pressure of $\pm 40 \mathrm{mmHg}$ (i.e., pressure between 80 and $160 \mathrm{mmHg}$ ) was applied to the inner surface of the artery for a total of three cycles to simulate a case of isolated systolic hypertension, which has been reported to be the most frequent form of hypertension in patients suffering from PAD. ${ }^{27}$

Blood flow computations investigated the effects of different oversizing ratios and the performances of both stents in a patient-specific artery. Following stent deployment, the deformed meshes of the artery and the stents were exported from Abaqus as open file formats (.obj) and converted to surface stl models using Solidworks 2013 (Dassault Systemes, Simulia Corp.,
RI, USA). These geometries were used for the generation of the computational volumetric grids, consisting of approximately 4.5 million tetrahedral elements, in ANSYS ICEM CFD (ANSYS Inc., Pittsburg, PA). The computational domain is adequately refined near the stent struts and the arterial wall. Through multiple refinements of the spatial mesh, different sizes of the computational grid are tested in a steady-state solution to ensure that the results are independent of the applied spatial discretization scheme. The analyses were performed with the finite volume code CFX 14.0 (ANSYS Inc., Pittsburg, PA), where the following continuity and Navier-Stokes equations are discretized with second order accuracy in space and time:

$$
\begin{aligned}
\nabla \cdot u & =0, \\
\rho \frac{\partial u}{\partial t}+\rho u \cdot \nabla u & =-\nabla p+\mu \nabla^{2} u,
\end{aligned}
$$

where $u$ is velocity $(\mathrm{m} / \mathrm{s}), t$ is time $(\mathrm{s}), \rho$ is density $\left(\mathrm{kg} / \mathrm{m}^{3}\right)$ and $p$ and $\mu$ are pressure (Pa) and dynamic viscosity (Pa s), respectively.

Physiological blood flow was simulated as a transient flow condition by applying the MRI-measured volumetric flow rate at the inlet of the artery (Fig. 6). ${ }^{36}$ 
The artery outlet was set to have zero pressure and a no-slip boundary condition was prescribed at the arterial wall and stent struts. Consequently, the outlet was extended enough to minimize the effects of boundary conditions on the accuracy of the simulations. As the plug-flow profile is applied at the inlet, it was also extended to reach a realistic blood flow profile. This way, a fully developed parabolic flow profile was guaranteed. Blood was modeled as an incompressible Newtonian fluid with a density of $1050 \mathrm{~kg} / \mathrm{m}^{3}$ and a viscosity of $3.5 \times 10^{-3} \mathrm{~Pa} \mathrm{~s}^{19}$ Three cardiac cycles were calculated using a time step size $0.01 \mathrm{~s}$. Each time step was assumed converged, when residuals reduced to $10^{-6}$ of their initial values.

A relation between restenosis and arterial stresses has previously been suggested, with high arterial stresses acting as a predictor of restenosis. ${ }^{38}$ On the other hand, the lack of an exact stress threshold that marks the beginning of such a process would only lead to indirect suggestions for a safe oversizing ratio. A better approach would be to define an oversizing limit based on tissue failure, which could be correlated by experimental findings. ${ }^{13}$ As a result, the maximum circumferential stresses in each layer were compared with their respective ultimate experimental stresses to specify the most failure-prone layer in the artery. Subsequently, the maximum circumferential stresses in this layer were evaluated to establish a correspondence between arterial stresses and unconstrained stent diameters.

The application of cyclic blood pressures following deployment provided the means to conduct fatigue analyses on the stents. The main aim was to calculate the factor of safety $\left(F O S_{\text {Nitinol }}\right)$ of the Nitinol stents, which represented the structural integrity of the implanted device. Since Nitinol is strain driven, mean strains and strain amplitudes were calculated at all integration points. Using the strain amplitude obtained from the 3rd cycle and a Nitinol endurance limit of $0.4 \%,{ }^{21} F O S_{\text {Nitinol }}$ was determined as:

$$
\frac{1}{F O S_{\text {Nitinol }}}=\frac{0.4}{\varepsilon_{\mathrm{amp}}}
$$

where $\varepsilon_{\text {amp }}$ represents the maximum strain amplitude and was calculated as the difference of the maximum principal strains between the systolic and diastolic blood pressures. A FOS value below +1 corresponds to material failure.

Spatial and temporal variations of arterial endothelial WSS are believed to play a critical role in hemodynamics of intact and stented arteries. ${ }^{25}$ WSS parameters such as time-average WSS (TAWSS) and oscillatory shear index (OSI) were derived from the numerically-calculated transient WSS field using an in-house MATLAB script and visualized by Tecplot (Tecplot Inc., Bellevue, WA). To obtain results independent of the temporal cycles and simulation initialization, the data of only the last cardiac cycle was evaluated. TAWSS, as a crucial factor to evaluate the hemodynamic status of the stented artery, was defined as $^{14}$ :

$$
\text { TAWSS }=\frac{1}{T} \int_{0}^{T}\left|\vec{\tau}_{w}\right| \mathrm{d} t,
$$

where $\left|\vec{\tau}_{w}\right|$ is the CFD-calculated magnitude of the instantaneous WSS vector $(\mathrm{Pa})$ and $T$ is the duration of one cardiac cycle (s). To include the effect of transient oscillation of the blood flow, OSI was defined as ${ }^{14}$ :

$$
O S I=\frac{1}{2}\left(1-\frac{\left|\int_{0}^{T} \vec{\tau}_{w} \mathrm{~d} t\right|}{\int_{0}^{T}\left|\vec{\tau}_{w}\right| \mathrm{d} t}\right) .
$$

To provide a less-sensitive hemodynamic performance indicator, the surface area exposed to low TASSW and high OSI was normalized with respect to the luminal surface area.

\section{RESULTS}

The preliminary simulations have been performed to validate the numerical models proposed for the artery and the Nitinol stents. The compliance of the arterial tract calculated with the model was $7.1 \%$ $\mathrm{mmHg}{ }^{-1} \times 10^{-2}$ which corresponded to experimental measurements acquired on the popliteal artery of subjects without evidence of PAD. ${ }^{35}$ Simulations of the arterial tissues showed a good agreement with uniaxial traction experiments conducted on each individual arterial layer (Fig. 2) ${ }^{13}$ In addition, the radial forces of the stents calculated with the model corresponded to the forces measured experimentally (Fig. 2). Furthermore, mechanical comparison of the two stents revealed that the Astron had a higher radial force compared to the Astron-Pulsar during its expansion from 1.8 to $7 \mathrm{~mm}$, thereby making it stiffer at the point of contact (about $5 \mathrm{~mm}$ ) with the artery (Fig. 2).

Following stent deployment, results indicated that adventitia was the arterial layer supporting most of the mechanical load. Moreover, adventitia was the most failure-prone layer, reaching up to $60 \%$ of its ultimate stresses ${ }^{13}$ for a normal-sized stent and up to $100 \%$ of the ultimate stresses when the oversizing ratio was 1.8 . For all cases, the maximum circumferential stresses in this layer increased non-linearly with respect to the unconstrained diameters of the Astron-Pulsar stent (Fig. 3). Furthermore, the relationship between acute luminal gain and stent diameters was also observed to 

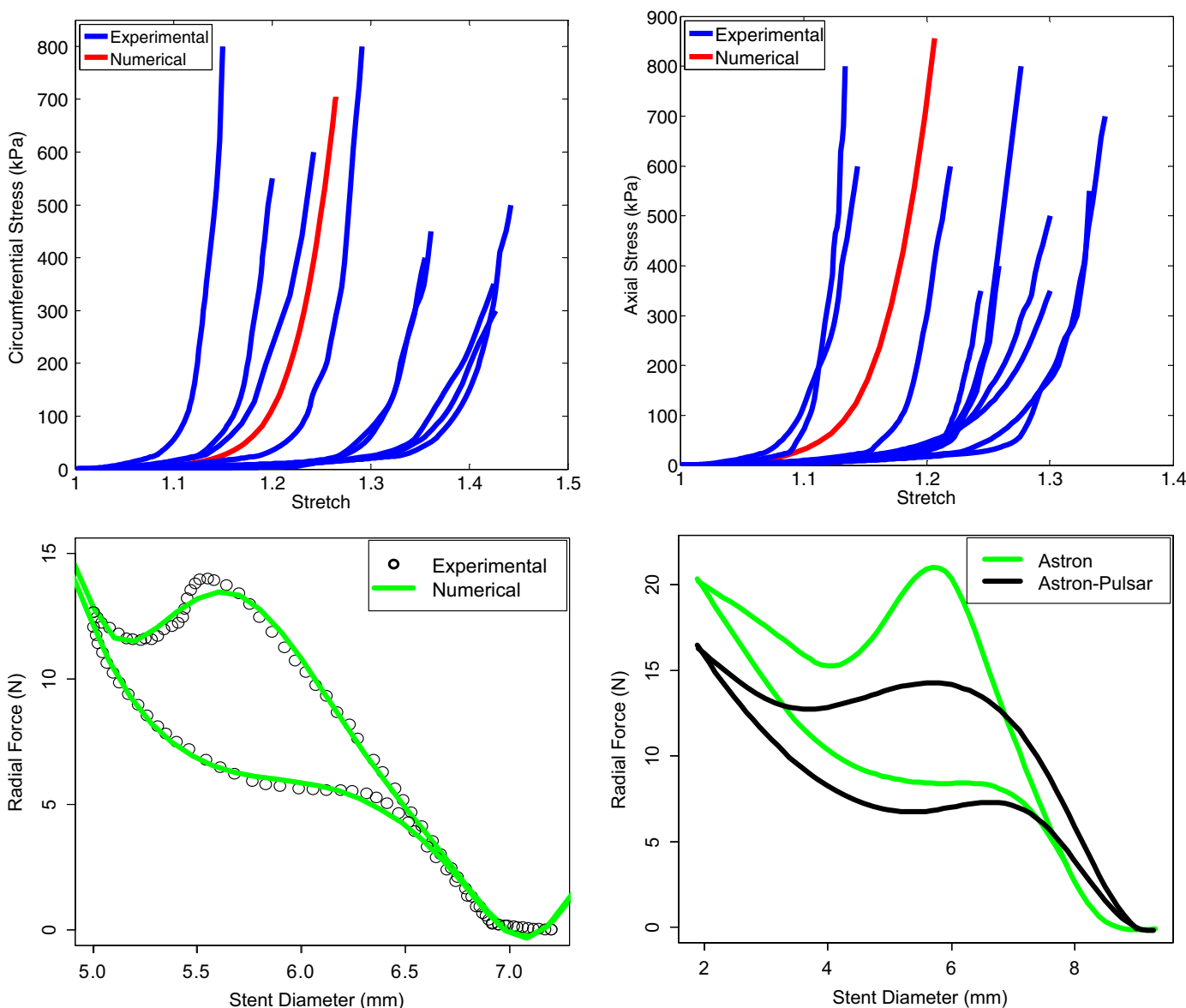

FIGURE 2. Uniaxial extension tests of the adventitial layer excised in circumferential (top left) and axial (top right) directions are numerically modeled and the resultant stress-strain relationships (red) are found to be in range of the diverse mechanical behaviors obtained via experiments (blue). The numerically obtained radial forces (green) of the Astron-Pulsar stent correspond to the experimental measurements (black) (bottom left). Additionally, the comparison of the radial forces of Astron-Pulsar (black) and Astron (green) stents highlights the stiffer nature of the Astron stent (bottom right).

be non-linear. Thus, when stents of 5.5,6,7 and $9 \mathrm{~mm}$ diameters were implanted, acute luminal gains were reported to be $7,10,11$ and $11.5 \%$, respectively (Fig. 3).

The non-linear increase in arterial stresses and lumen gains was similar for both stents in all arteries (Fig. 4). Due to its higher radial force, the Astron stent produced higher stresses in the arteries when compared with the Astron-Pulsar stent at each pressure level (Fig. 4). In the straight arterial model, the maximum difference between the stresses produced by the two stents was about $30 \%$ for both normal-sized and oversized stents. This difference increased to more than $40 \%$ in patient-specific arteries (Fig. 4). The artery also had a greater expansion with the Astron stent, in which the maximum increase of the lumen gain was 1.5 and $2.5 \%$ for straight and patient-specific arteries, respectively (Fig. 4).

Regardless of the arterial geometry, the fatigue behavior of the Astron-Pulsar stent showed similar behaviors. Increasing the unconstrained stent diameters led to an increase in the mean strains at the integration points; with the average maximum values being reported as $1.70 \pm 0.51,3.38 \pm 0.89,6 \pm 0.07$ and $7.07 \% \pm 0.11 \%$ for $5.5,6,7$ and $9 \mathrm{~mm}$, respectively (Fig. 5). In contrast, there was a smaller increase in the strain amplitude with increasing oversizing ratios. Consequently, the factor of safety of the stent was found as $5.28 \pm 0.1,4.94 \pm 0.11,3.64 \pm 0.27$ and $1.18 \pm 0.11$ for $5.5,6,7$ and $9 \mathrm{~mm}$, respectively. A similar gradual decrease in the safety factor of the stent was observed for the Astron as well.

For all deployment cases and for both stent models, spatial structure of blood flow and instantaneous patterns of WSS were found to be complex and varying during the cardiac cycle (Fig. 6). Throughout the cardiac cycle and for the time-integrated parameter, localized areas of atheroprone low WSS (less than 5\% of maximum WSS i.e., $<0.5 \mathrm{~Pa}$ ) were seen in regions of flow separation, e.g., near the stent struts and inner 

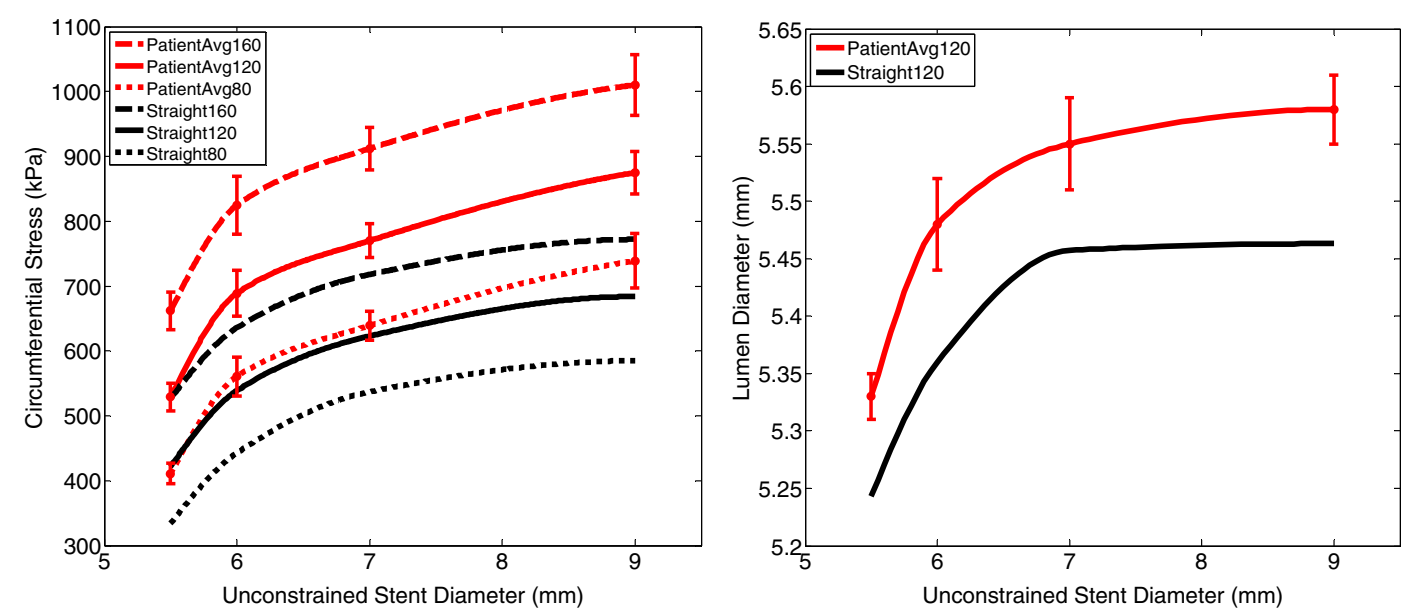

FIGURE 3. The average maximum arterial circumferential stresses of the patient-specific arteries (red) exceed the stress levels observed in a straight artery (black) and show a less emphasized non-linear behavior for all blood pressure values (range: 80-160 $\mathrm{mmHg}$ ) (left). Regardless of the arterial geometries, a very small lumen gain is achieved by oversizing the stents (right).
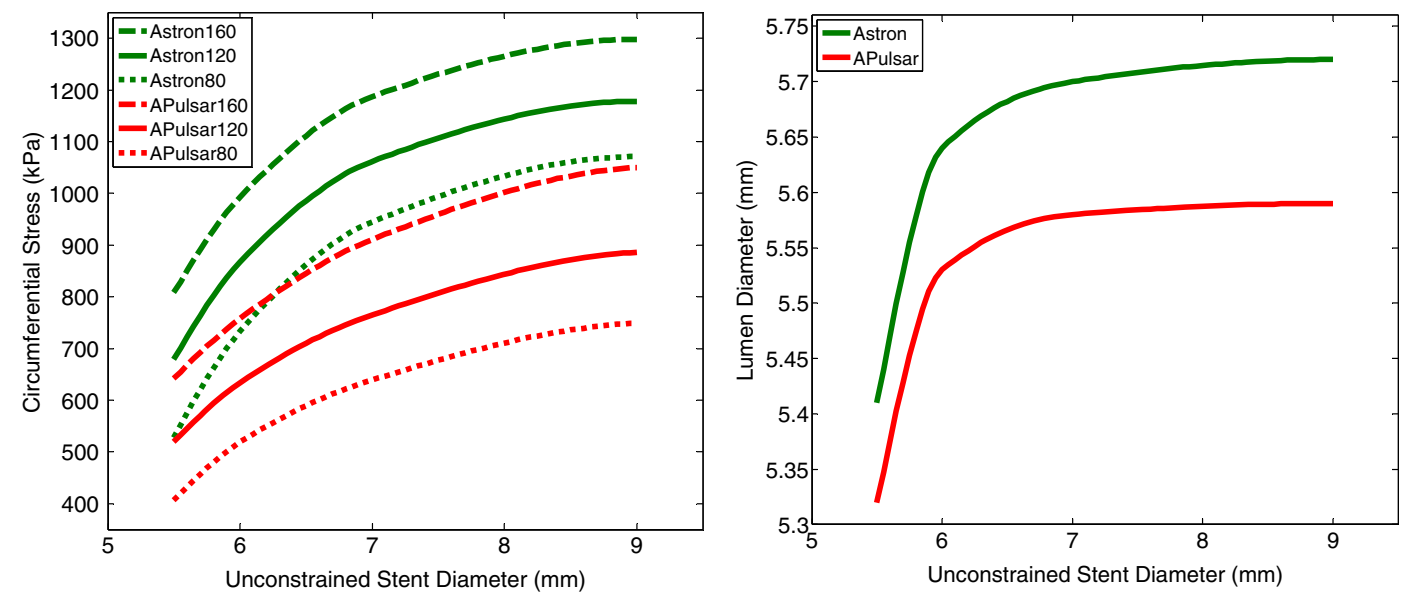

FIGURE 4. The stent with a higher radial force, Astron (green), was found to create significantly higher stress levels than the Astron-Pulsar stent (red) (left). In comparison, a very limited increase in lumen gain is observed to create a mismatch in arterial stress-lumen relationship (right). Regardless of the stent designs, a very small lumen gain is achieved by oversizing the stents (right).

side of curved artery. Regions of high WSS, on the other hand, were likely to occur on the outer side of the arterial curvature and on the in-lumen-protruded side of the stent struts. Independent of the stent stiffness, length and geometry, larger areas were exposed to low WSS at proximal portions of the stented segments. Furthermore, the distribution of OSI, as a measure of temporal WSS change, in both stents showed similar traits with the TAWSS distribution. High values of OSI (more than $20 \%$ of maximum OSI i.e., $>0.1$ ) were seen in the vicinity of stent struts, inter-strut connectors, proximal segment of the stented artery and distal inner wall of the curved artery.

A direct comparison of all cases showed that increasing the stent-induced arterial diameter would raise the normalized surface area exposed to low
TAWSS (less than $0.5 \mathrm{~Pa}$ ), which was concurrently more pronounced for the cases stented with the Astron (Fig. 7). In addition, the deployment of Astron definitively assured that the surface area exposed to high atheroprone values of OSI would be bigger than with the Astron-Pulsar (Fig. 7). No clear relation between the oscillatory features of blood flow and different lateral expansion rates was observed.

\section{DISCUSSION}

Nitinol stent oversizing is commonly performed during endovascular procedures; however, currently, stent sizing mostly relies on the expertise of the clinicians and is defined by stent manufacturers and not by 

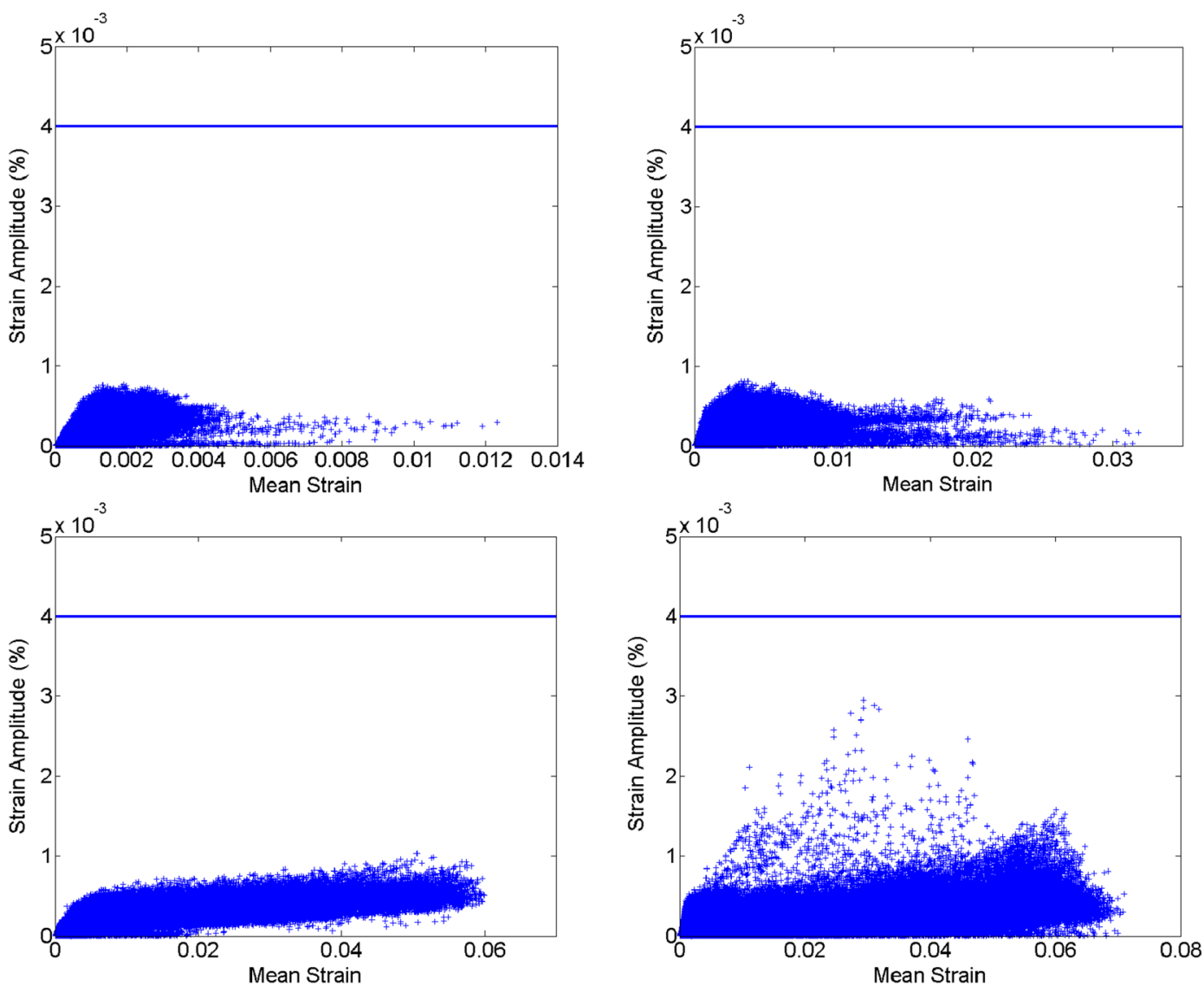

FIGURE 5. The fatigue behavior of the Pulsar stent when deployed into a geometrically accurate artery with unconstrained stent diameters of $5 \mathrm{~mm}$ (top left), $6 \mathrm{~mm}$ (top right), $7 \mathrm{~mm}$ (bottom left) and $9 \mathrm{~mm}$ (bottom right): As the oversizing ratio is increased, the strain amplitude of more points are found to migrate towards the Nitinol safety limit of 0.4 ; thereby decreasing the safety factor of the stent.

objective criteria. While oversizing a stent will undoubtedly increase acute luminal gain, it can also create a hostile chronic environment for the artery through stent-to-arterial wall interactions, which may ultimately lead to arterial damage, neointimal/adventitial hyperplasia and restenosis. ${ }^{28,41}$ With previous clinical studies reporting controversial findings on the subject, ${ }^{3,16,22,28}$ the efficacy of oversizing remains an issue to be answered. Thus, to improve the understanding of mechanical effects of stent oversizing in the popliteal arterial tract, numerical analyses have been performed within the present investigation.

The hypothesis behind stent oversizing is that oversized stents will limit possible arterial restenosis due to acute lumen enlargement. However, results of this study showed a non-linear relationship between the lumen gain and oversizing ratio (Figs. 3, 4). Increased oversizing led to a small lumen increase, while generating rapidly increasing arterial stresses (Figs. 3, 4). This mismatch between the limited lumen gain and elevating stresses can be illustrated by a $3 \%$ lumen vs. $30 \%$ stress increase for the Astron-Pulsar stent ( 4 vs. $33 \%$ for the Astron) when the stent-toartery ratio is transitioned from 1.1 to 1.2. Therefore, our results suggest that instead of providing an additional lumen gain, stents with higher oversizing ratios inflict greater chronic arterial damages through increased chronic arterial stresses.

A comparison between the numerical and experimental results indicated that with increased oversizing, the level of arterial stresses came close to the failure limits of the tissues, which have been obtained via uniaxial extension tests. ${ }^{13}$ Independent of the stent design, adventitia was found to be the most vulnerable layer due to its highly nonlinear mechanical response at pressures above $80 \mathrm{mmHg} .{ }^{32}$ The high risk of failure in this layer further suggests that normal sized stents are more favorable to protect arterial walls from chronic damage and, consequent, neoadventitial hyperplasia. ${ }^{32}$

Results of the numerical calculations showed that both the maximum arterial circumferential stresses and the luminal gains increased non-linearly with respect to 
(a)

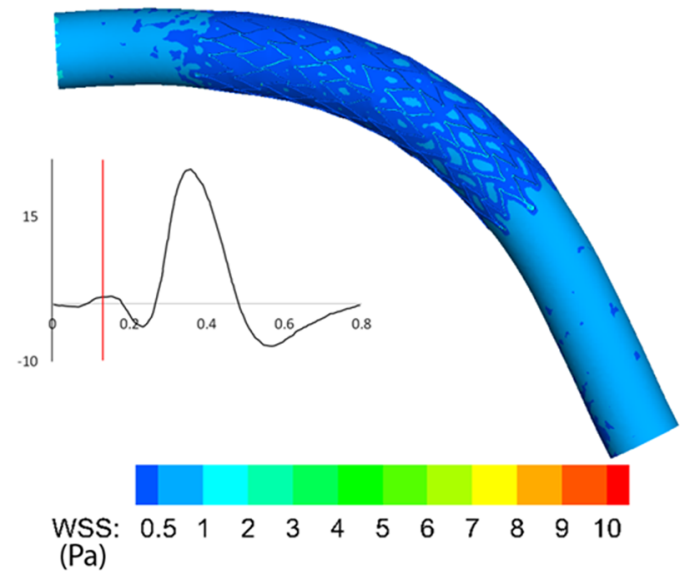

(c)

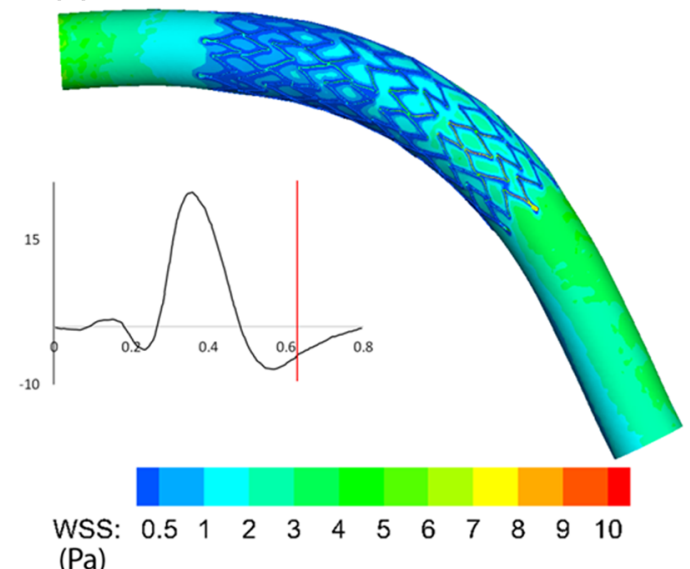

(b)

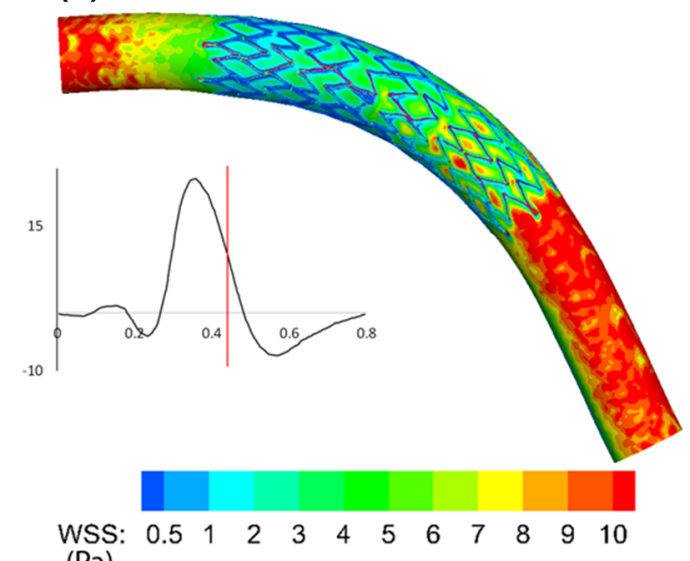

$(\mathrm{Pa})$

(d)

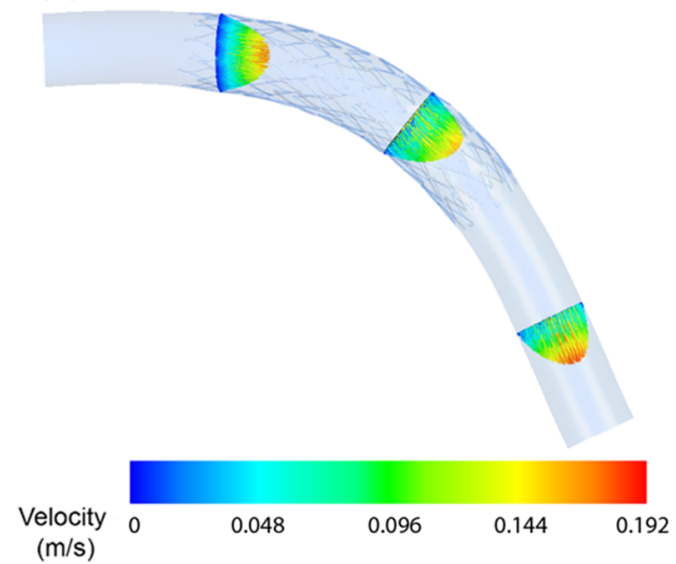

FIGURE 6. Instantaneous pattern of wall shear-stress (WSS) distribution on stented popliteal artery at different instants of a cardiac cycle marked by solid red line on a flow rate profile inset. WSS is shown at: (a) $t=0.13 \mathrm{~s}$ (b) $t=0.44 \mathrm{~s}$ and (c) $t=0.64 \mathrm{~s}$. Panel (d) shows how the symmetric velocity profile is distorted toward the outer wall owing to arterial curvature. This affects the WSS distribution considerably.

the unconstrained stent diameters regardless of arterial geometries and stent designs (Figs. 3, 4). Comparisons between straight and patient-specific geometries showed that the latter exhibited a slightly less pronounced non-linear increase of the mechanical parameters with increased oversizing. This may be explained by distinctive deformations exerted onto different arterial geometries during stent deployment. While the straight artery was only displaced radially, the curved arteries were subjected to straightening alongside radial expansion. This effect not only caused an overall increase of the circumferential stresses for each oversizing ratio, but also led to higher stress differences between oversized and normal-sized arteries.

The successful outcome of an endovascular procedure not only lies in selecting the correct stent size, but also in selecting the correct stent design. In the present study, the disproportionate increase between lumen gain and arterial stresses for successive oversizing ratios was similar for both stents (Fig. 4). On the other hand, oversizing the Astron stent to the ratio of 1.2 increased the risk of failure considerably when compared with the Astron-Pulsar stent. Furthermore, a maximum difference of $2.5 \%$ was observed between the lumen gains achieved at this oversizing ratio (Fig. 4). This value remains small when compared with their corresponding stress difference of $37 \%$ (Fig. 4). Therefore, this mismatch between the changes in the stresses and lumen diameters suggests that radial force would have an impact on the processes observed during clinical follow-up, as stents with higher radial forces would likely inflict more chronic irritation to arterial walls when compared with their less stiff counterparts.

Individual patient anatomies in the present study all showed different curvatures. However, no relation between the fatigue behavior of either the AstronPulsar or the Astron and arterial geometries was 

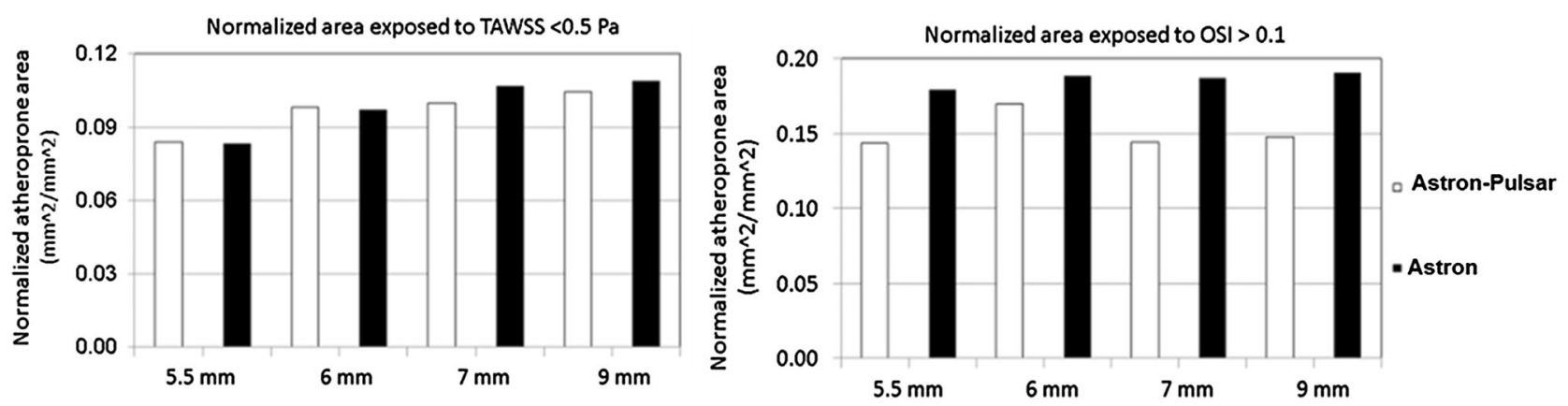

FIGURE 7. Normalized surface area exposed to time-averaged wall shear stress (TAWSS) of less than $0.5 \mathrm{~Pa}$ (left) and oscillatory shear index (OSI) of more than 0.1 (right). Oversizing leads to larger areas being exposed to low TAWSS and more prominent atheroprone conditions at the arterial wall, which is more noticeable for the cases with the stiffer Astron stent. On the other hand, while the deployment of Astron noticeably rises the oscillatory characteristics of local blood flow, those features are not directly affected by stent oversizing.

observed. Instead, the stents performed similarly for all curved arteries. This may be explained by the fact that an identical systolic-diastolic pressure behavior was applied to all models. ${ }^{24}$ The safety factor of the Astron-Pulsar stent was found to decrease with increasing oversizing ratio, which can be explained by the higher deformations exhibited by oversized stents due to their incomplete expansions at deployment. Although the calculated fatigue criterion remained below the failure level, stents with $9 \mathrm{~mm}$ diameters came close to the Nitinol endurance limit of $0.4 \%$ (Fig. 5), ${ }^{21}$ without even considering the mechanical forces acting on the stent due to the physiological loading present in lower limb arteries. ${ }^{9}$ This result suggests that oversizing increases the risks of stent failures, which has been directly related with restenosis. ${ }^{17}$

Physiological stressors such as near-wall flow, which directly perturb intra- and extracellular signaling, proved to be a reliable predictor for local biological responses of stented arteries. ${ }^{2}$ Thus, studying the hemodynamic performance of implanted stents and considering procedural variabilities, such as oversizing, could be used to provide clinicians with accurate observations on the causes of adverse biological effects. Local flow patterns are proved to affect the neointimal formation, in-stent restenosis and thrombosis in stented arteries. Therefore, computed indices of flow were calculated here to support the hypothesized drawback of stent oversizing. The critical values of low WSS and high OSI, which are reported to correlate with adverse clinical outcomes, ${ }^{25}$ were found to be similar to already published studies. ${ }^{26}$ Stent oversizing considerably perturbed the local flow patterns, specifically the near-wall flow, due to the abrupt change in the proximate bounding geometry. The procedure was found to increase the normalized surface area exposed to low TAWSS, leading to a large area of the stented artery to be susceptible to unfavorable biological responses. The abrupt change of arterial size and the expanded cross-section would decrease the blood flow and the WSS in the vicinity of the stented wall (Fig. 6). For cases where significant lumen gain is not achieved, this would depress the hemodynamic efficiency of the oversized stent and seriously question its clinical expediency. In contrast, the larger surface area exposed to high OSI was not affected by the stent-to-artery ratio (Fig. 7). This may be mainly related to the specific shape of the stent and curvature of the artery, wherein the secondary flow generated by the arterial curvature and recirculation zones induced by the stent will have a combinatory effect on the oscillatory characteristics of the blood flow. For the cases implanted with the Astron stent, larger deformations were induced in the artery walls around the stent struts, which resulted in a larger alteration of near-wall flow compared to the more flexible Astron-Pulsar stent. Consequently, computed indices of flow such as velocity and WSS are more affected when stiffer stents are used. In conclusion, stent-induced oversizing of the arterial wall, independent of the stent type, would deteriorate the hemodynamic performance of peripheral stents, wherein the arteries fitted with stiffer stents become more vulnerable.

The validity of the proposed arterial model was verified by comparing the numerical simulations with published experimental data. The individual behaviors of each arterial layer obtained via traction simulations, as well as the overall compliance of the artery, matched well with existing measurements (Fig. 2). ${ }^{13,35}$ Furthermore, the mechanical behavior of the adventitia is similar to previous reports, ${ }^{32}$ as it became the mechanically predominant layer when the physiological blood pressure of $13.3 \mathrm{kPa}$ is exceeded. These comparisons indicated that the present arterial model 
is appropriate to evaluate arterial stenting. Finally, the immediate lumen gain values obtained from the deployment simulations were compared with a clinical investigation that performed Nitinol stent oversizing in the femoral arteries of Yucatan swines. ${ }^{41}$ These clinical findings compared favorably with the lumen gains obtained in our study for high oversizing ratios ( $>1.4)$. On the other hand, numerical models with lower oversizing ratios $(<1.4)$ predicted a larger lumen gain, which can be attributed to the difference between the numerical and clinical arterial diameters prior to stenting.

Several limitations to the present study need to be discussed. Only healthy arteries were modeled and stented, while stents are generally implanted in diseased arteries. The inclusion of plaques into the model would not only change the stress/strain values, but would also have an impact on the location of maximum stress/strain concentrations in the arterial models. However, based on current understanding, deployed stents usually overlap with the nonobstructed parts of an artery, thereby exerting potential effects caused by stent oversizing on both healthy and diseased sections. Moreover, pre-clinical investigations have so far used healthy animal arteries to investigate the effects of oversizing. Thus, the results could easily be compared to validate the numerical models. Only two stent designs were used in this study. Although the effects of oversizing are not expected to change with different stent designs, the level of stress and lumen expansion may show differences. Performing similar calculations for different designs could work towards achieving an average value for a safe oversizing ratio. Since the present study focused on the parametric evaluation of the stenting procedure, the same material model was used for all patient-specific arteries. Due to this parametric approach, the conclusion on the effect of oversizing should remain identical for each patient, although the exact level of strain may differ. Finally, the residual strains for the arteries were not modeled, which may suggest a possible overestimation of the arterial stresses. Nonetheless, the nonlinear stress behavior with increasing stent diameters would be the same. The accuracy of blood flow simulations depends on the chosen boundary conditions. In order to obtain more realistic results, direct experimental measurements should be used to define the boundary conditions for blood velocity or pressure at the inlet and outlet. In this study, a generic dataset obtained from an MRI measurement was applied at the inlet of the domain. However, a simplified boundary condition was used at the outlet with a zero pressure. To limit the error introduced by this approximation, the outlet was extended to maintain realistic flow profiles.
The effects of Nitinol stent oversizing in geometrically patient-specific popliteal arteries were evaluated through numerical analyses. A gradual increase of the stent-to-artery ratio from 1.1 to 1.8 showed the development of both the structural and hemodynamic properties of arteries with respect to the normal and oversized stent diameters, while providing the framework for fatigue analyses of the stents. The results showed that oversizing had a very limited impact on the immediate lumen gains achieved following stenting. At the same time, transition to a higher oversizing ratio led to a significant increase in structural and WSS in arteries, which can be associated with arterial damage and disruption in the blood flow. Since selfexpanding stents will continuously apply radial forces to the arterial walls in order to reach their nominal diameters, these damages will continue to accumulate, creating a mechanical environment prone to induce restenosis. Therefore, to increase the success rate of an endovascular procedure in a FP arterial tract that includes healthy popliteal segments, clinicians should take care not to oversize Nitinol stents and allow them to contribute to a late lumen gain in the arteries.

\section{ACKNOWLEDGMENTS}

This investigation was supported by the Research Council of the Kantonsspital Aarau, the Swiss Heart Foundation, the Gotthard Schettler Foundation and the Swiss National Science Foundation. The authors have no commercial, proprietary, or financial interest in any products or companies described in this article.

\section{REFERENCES}

${ }^{1}$ Cha, S.-H., M. H. Han, Y. H. Choi, C. J. Yoon, S. K. Baik, S. J. Kim, and K. H. Chang. Vascular responses in normal canine carotid arteries. Invest. Radiol. 38:95-101, 2003.

${ }^{2}$ Chatzizisis, Y. S., A. U. Coskun, M. Jonas, E. R. Edelman, C. L. Feldman, and P. H. Stone. Role of endothelial shear stress in the natural history of coronary atherosclerosis and vascular remodeling. molecular, cellular, and vascular behavior. J. Am. Coll. Radiol. 49:2379-2393, 2007.

${ }^{3}$ Chen, H. Y., A. K. Sinha, J. S. Choy, H. Zheng, M. Sturek, B. Bigelow, D. L. Bhatt, and G. S. Kassab. Mis-sizing of stent promotes intimal hyperplasia: impact of endothelial shear and intramural stress. AJP Heart Circ. Physiol. 301:H2254-H2263, 2011.

${ }^{4}$ Chiastra, C., S. Morlacchi, D. Gallo, U. Morbiducci, R. Cárdenes, I. Larrabide, and F. Migliavacca. Computational fluid dynamic simulations of image-based stented coronary bifurcation models. $J$. R. Soc. Interface 10:20130193, 2013. 
${ }^{5}$ Conway, C., J. P. McGarry, and P. E. McHugh. Modelling of atherosclerotic plaque for use in a computational testbed for stent angioplasty. Ann. Biomed. Eng. 42:2425-2439, 2014.

${ }^{6}$ Early, M., C. Lally, P. J. Prendergast, and D. J. Kelly. Stresses in peripheral arteries following stent placement: a finite element analysis. Comput. Methods Biomech. Biomed. Eng. 12:25-33, 2009.

${ }^{7}$ Elsheikh, A., C. Whitford, R. Hamarashid, W. Kassem, A. Joda, and P. Büchler. Stress free configuration of the human eye. Med. Eng. Phys. 35:211-216, 2013.

${ }^{8}$ Gasser, T. C., R. W. Ogden, and G. A. Holzapfel. Hyperelastic modelling of arterial layers with distributed collagen fibre orientations. J. R. Soc. Interface 3:15-35, 2006.

${ }^{9}$ Gökgöl, C., N. Diehm, and P. Büchler. Quantification of deformation of the femoro-popliteal arterial tract during leg flexion in subjects with peripheral arterial disease. J. Endovasc. Ther. 20:825-835, 2013.

${ }^{10}$ Gornik, H. L., and J. A. Beckman. Cardiology patient page. Peripheral arterial disease. Circulation 111:e169-e172, 2005.

${ }^{11}$ Hoffmann, R., G. S. Mintz, J. J. Popma, L. F. Satler, A. D. Pichard, K. M. Kent, C. Walsh, P. Mackell, and M. B. Leon. Chronic arterial responses to stent implantation: a serial intravascular ultrasound analysis of Palmaz-Schatz stents in native coronary arteries. J. Am. Coll. Cardiol. 28:1134-1139, 1996.

${ }^{12}$ Holzapfel, G. A. Mechanics of Angioplasty: wall, balloon and stent. In: Mechanics in Biology, AMD-Vol. 242/BEDVol. 46, edited by J. Casey, and G. Bao. New York: The American Society of Mechanical Engineers, 2000, pp. 141156.

${ }^{13}$ Holzapfel, G. A., G. Sommer, and P. Regitnig. Anisotropic mechanical properties of tissue components in human atherosclerotic plaques. J. Biomech. Eng. 126:657-665, 2004.

${ }^{14}$ Huo, Y., T. Wischgoll, and G. S. Kassab. Flow patterns in three-dimensional porcine epicardial coronary arterial tree. Am. J. Physiol. Heart Circ. Physiol. 293:H2959-H2970, 2007.

${ }^{15}$ Katritsis, D. G., A. Theodorakakos, I. Pantos, M. Gavaises, N. Karcanias, and E. P. Efstathopoulos. Flow patterns at stented coronary bifurcations: computational fluid dynamics analysis. Circ. Cardiovasc. Interv. 5:530 539, 2012.

${ }^{16}$ Kirsch, E. C., M. S. Khangure, P. Morling, T. J. York, and W. McAuliffe. Oversizing of self-expanding stents : influence on the development of neointimal hyperplasia of the carotid artery in a canine model. Am. J. Neuroradiol. 23:121-127, 2002.

${ }^{17}$ Kröger, K., F. Santosa, and M. Goyen. Biomechanical incompatibility of popliteal stent placement. J. Endovasc. Ther. 11:686-694, 2004

${ }^{18}$ LaDisa, J. F., L. E. Olson, I. Guler, D. A. Hettrick, J. R. Kersten, D. C. Warltier, and P. S. Pagel. Circumferential vascular deformation after stent implantation alters wall shear stress evaluated with time-dependent 3D computational fluid dynamics models. J. Appl. Physiol. 98:947-957, 2005.

${ }^{19}$ Milnor, W. R. Hemodynamics. Baltimore: Williams \& Wilkins, 1989.

${ }^{20}$ Mortier, P., G. A. Holzapfel, M. De Beule, D. Van Loo, Y. Taeymans, P. Segers, P. Verdonck, and B. Verhegghe. A novel simulation strategy for stent insertion and deploy- ment in curved coronary bifurcations: comparison of three drug-eluting stents. Ann. Biomed. Eng. 38:88-99, 2010.

${ }^{21}$ Pelton, A. R., V. Schroeder, M. R. Mitchell, X.-Y. Gong, M. Barney, and S. W. Robertson. Fatigue and durability of Nitinol stents. J. Mech. Behav. Biomed. Mater. 1:153-164, 2008.

${ }^{22}$ Piamsomboon, C., G. S. Roubin, M. W. Liu, S. S. Iyer, A. Mathur, L. S. Dean, C. R. Gomez, J. J. Vitek, N. Chattipakorn, and G. Yates. Relationship between oversizing of self-expanding stents and late loss index in carotid stenting. Cathet. Cardiovasc. Diagn. 143:139-143, 1998.

${ }^{23}$ Poon, E. K. W., P. Barlis, S. Moore, W.-H. Pan, Y. Liu, Y. Ye, Y. Xue, S. J. Zhu, and A. S. H. Ooi. Numerical investigations of the haemodynamic changes associated with stent malapposition in an idealised coronary artery. J. Biomech. 47:2843-2851, 2014.

${ }^{24}$ Rebelo, N., R. Fu, and M. Lawrenchuk. Study of a nitinol stent deployed into anatomically accurate artery geometry and subjected to realistic service loading. J. Mater. Eng. Perform. 18:655-663, 2009.

${ }^{25}$ Rikhtegar, F., J. A. Knight, U. Olgac, S. C. Saur, D. Poulikakos, W. Marshall, P. C. Cattin, H. Alkadhi, and V. Kurtcuoglu. Choosing the optimal wall shear parameter for the prediction of plaque location-A patient-specific computational study in human left coronary arteries. Atherosclerosis 221:432-437, 2012.

${ }^{26}$ Rikhtegar, F., F. Pacheco, C. Wyss, K. S. Stok, H. Ge, R. J. Choo, A. Ferrari, D. Poulikakos, R. Müller, and V. Kurtcuoglu. Compound ex vivo and in silico method for hemodynamic analysis of stented arteries. PLoS ONE 8:e58147, 2013.

${ }^{27}$ Safar, M. E., P. Priollet, F. Luizy, J.-J. Mourad, P. Cacoub, H. Levesque, J. Benelbaz, P. Michon, M. Herrmann, and J. Blacher. Peripheral arterial disease and isolated systolic hypertension: the ATTEST study. J. Hum. Hypertens. 23:182-187, 2009.

${ }^{28}$ Saguner, A. M., T. Traupe, L. Räber, N. Hess, Y. Banz, A. R. Saguner, N. Diehm, and O. M. Hess. Oversizing and restenosis with self-expanding stents in iliofemoral arteries. Cardiovasc. Intervent. Radiol. 35:906-913, 2012.

${ }^{29}$ Scheinert, D., S. Scheinert, J. Sax, C. Piorkowski, S. Bräunlich, M. Ulrich, G. Biamino, and A. Schmidt. Prevalence and clinical impact of stent fractures after femoropopliteal stenting. J. Am. Coll. Cardiol. 45:312-315, 2005.

${ }^{30}$ Schillinger, M., S. Sabeti, P. Dick, J. Amighi, W. Mlekusch, O. Schlager, C. Loewe, M. Cejna, J. Lammer, and E. Minar. Sustained benefit at 2 years of primary femoropopliteal stenting compared with balloon angioplasty with optional stenting. Circulation 115:2745-2749, 2007.

${ }^{31}$ Schillinger, M., S. Sabeti, C. Loewe, P. Dick, J. Amighi, W. Mlekusch, O. Schlager, M. Cejna, J. Lammer, and E. Minar. Balloon angioplasty versus implantation of nitinol stents in the superficial femoral artery. N. Engl. J. Med. 354:1879-1888, 2006.

${ }^{32}$ Schulze-bauer, C. A. J., P. Regitnig, and G. A. Holzapfel. Mechanics of the human femoral adventitia including the high-pressure response. Am. J. Physiol. Heart. Circ. Physiol. 282:2427-2440, 2002.

${ }^{33}$ Seo, T., L. G. Schachter, and A. I. Barakat. Computational study of fluid mechanical disturbance induced by endovascular stents. Ann. Biomed. Eng. 33:444-456, 2005.

${ }^{34}$ Stiegler, H., and R. Brandl. Importance of ultrasound for diagnosing periphereal arterial disease. Ultraschall Med. 30:334-374, 2009. 
${ }^{35}$ Tai, N. R., A. Giudiceandrea, H. J. Salacinski, A. M. Seifalian, and G. Hamilton. In vivo femoropopliteal arterial wall compliance in subjects with and without lower limb vascular disease. J. Vasc. Surg. 30:936-945, 1999.

${ }^{36}$ Thompson, R. B., and E. R. Mcveigh. Real-time volumetric flow measurements with complex-difference MRI. Magn. Reson. Med. 50:1248-1255, 2008.

${ }^{37}$ Timmins, L. H., C. A. Meyer, M. R. Moreno, and J. E. Moore. Effects of stent design and atherosclerotic plaque composition on arterial wall biomechanics. J. Endovasc. Ther. 15:643-654, 2008.

${ }^{38}$ Timmins, L. H., M. W. Miller, F. J. Clubb, and J. E. Moore. Increased artery wall stress post-stenting leads to greater intimal thickening. Lab. Invest. 91:955-967, 2011.
${ }^{39} \mathrm{Wu}$, W., W.-Q. Wang, D.-Z. Yang, and M. Qi. Stent expansion in curved vessel and their interactions: a finite element analysis. J. Biomech. 40:2580-2585, 2007.

${ }^{40}$ Zhao, S., L. Gu, and S. R. Froemming. Finite element analysis of the implantation of a self-expanding stent: impact of lesion calcification. J. Med. Device. 6:021001, 2012.

${ }^{41}$ Zhao, H. Q., A. Nikanorov, R. Virmani, R. Jones, E. Pacheco, and L. B. Schwartz. Late stent expansion and neointimal proliferation of oversized Nitinol stents in peripheral arteries. Cardiovasc. Intervent. Radiol. 32:720726, 2009. 